\title{
Multidirectional UV-LED lithography using an array of high-intensity UV-LEDs and tilt-rotational sample holder for 3-D microfabrication
}

\author{
Sabera Fahmida Shiba, Jun Ying Tan and Jungkwun Kim * (1)
}

\begin{abstract}
This paper presents a computer-controlled multidirectional UV-LED lithography system for 3-D microfabrication. The presented UV-LED system has adopted adjustable or programmable high-intensity UV-LEDs as a light source enabling for photopatterning both thin and thick SU-8 photoresist process. The prototype of the proposed system comprises 5-by-5 surface-mounted type LEDs with customized collimation lens, a tilt-rotational sample holder for introducing multidirectional light exposure, and a computer-control asset for synchronized controls of the light source and the sample holder. An adjustable light intensity provides an ease of lithography both for a few micron feature size patterning and a millimeter thick photopatterning by providing optimal light exposure condition by considering diffraction, and absorption of the UV light. Together with the tilt-rotational sample holder, the multidirectional UV-LED lithography can fabricate various 3-D microstructures in a wide range of the photoresist film thickness. The 3-D fabrications include an array of micro triangle slabs, 1-mm tall pillar array, the different scale same 3-D geometry structures on the same substrate.
\end{abstract}

Keywords: UV-LED lithography, 3D microfabrication, Millimeter thick photoresist process

\section{Introduction}

Ultra-violet (UV) lithography has been a fundamental nano/micro patterning process and widely used in the industry and the research institutes for the past decades as the fabrication process is relatively simple with high repeatability, which is important for manufacturability. Recent UV lithography has advanced for patterning three-dimensional (3-D) nano/micro fabrication by introducing a precise control of an automated mechanical system. Contact liquid photolithographic polymerization (CLiPP) demonstrated layer-by-layer photopatterning with a fine movement of the sample stage with $\mathrm{x}-\mathrm{y}-\mathrm{z}$ direction to form a 3-D microfluidic

*Correspondence: kjungkwun@gmail.com

Department of Electrical and Computer Engineering, Kansas State University, Manhattan, KS 66506, USA device [2]. In a similar way, a stereolithographic approach was also introduced for photopatterning of metal molding using a positive photoresist [3]. A focused UV light stayed at a fixed position with on-and-off function is synchronized with an $\mathrm{x}-\mathrm{y}-\mathrm{z}$ movable sample holder to draw a pattern by layers to form 3-D structure. A prismassisted lithography introduced to change the light exposure angle to photopattern a slanted sidewall of the 3-D microstructure [4]. A backside UV exposure and multiple layer stacking method by the aid of a fine alignment system was also introduced to form several hundred micron 3-D structures for cell culture scaffold application [12]. Maskless lithography methods using digital micromirror (DMD) and liquid crystal display (LCD) were also introduced and have become the basis of today's DMD or LCD 3-D printer [11, 13]. 
While a number of 3-D microlithography methods have utilized a layer-by-layer stage control to form a 3-D photopatternable structures, the nature of those fabrication methods is to have a number of layers which often prevent to have a smooth curved or inclined structures. Those sidewall stepping surfaces become more challenging to fabricate RF or optical microdevices as those multiple layered stepping structures are not suitable for. A multidirectional UV lithography has been introduced for 3-D microfabrication where a sample holder was tiltrotational to create smooth inclined 3-D microstructures with a single direct exposure $[1,14]$ as described in Fig. 1. Since the tilt-rotational sample holder could introduce various inclined angles tangent to the light source with a programmable control, various 3-D inclined structures such as horns, tilted pillar, and triangle shapes have been freely able to photopattern. This method was superior for the surface quality of inclined sidewall over the prism assisted lithography, or lay-by-layer lithography method. With an automated multidirectional lithography system, complex 3-D microstructures such as a letter horns and a ribbon horn [7], and nanoscale 3-D structures [5] were presented as advanced 3-D structures. Also, introducing various refractive index mediums in the automated multidirectional lithography extended 3-D projectable volume by increasing the tilt-able angle [10] and millimeter height pillar were introduced by increasing the exposure time by demonstrating 3-D toroid inductor [6].

After the multidirectional UV lithography scheme has adopted the UV-LEDs as its light source [9], the UV-LED based multidirectional UV lithography has continuously advanced including the features of the ease of individual LEDs switchings as well as UV exposure intensity variation from the recently updated report [8]. In this paper, higher light intensity and a low attenuation light

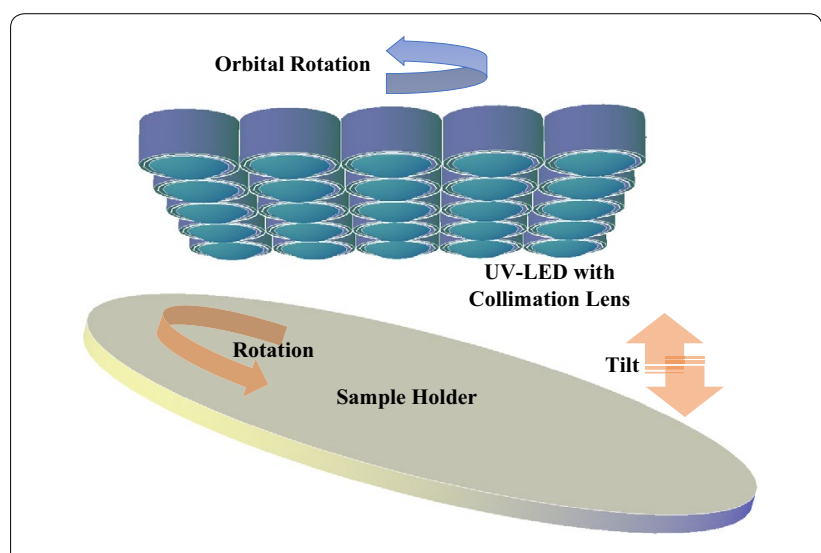

Fig. 1 Description of multidirectional UV-LED lithography collimation have advanced by adopting high-power surface mount type LEDs and an individual one-lens with light waveguide for light collimation. Also, an orbital rotation of the entire light source provides uniform light distribution while the regional control of the light exposure area is capable of creating various 3D microstructures. From the introduced system, the highest light intensity has been reported to be $200 \mathrm{~mW} / \mathrm{cm}^{2}$, which is higher than any commercial lithography system. With a wide range of variable light intensity as well as a programmable lighting feature from the array of LEDs introduce a single UV exposure for 3D microstructures which have a great potential for advanced sensors, RF/microwave microdevices such as $3 \mathrm{D}$ antennas or waveguide, and bioMEMS including microfluidic channels or drug delivery devices.

\section{System setup and characterization}

The proposed system comprises a light source, a tiltrotational stage, and a computer control part as presented in Fig. 2. The light source includes UV-LEDs, lens, optical waveguide, and a heat sink. The 405-nm (h-line) peak LEDs (LEDUVA35T01VL00, LG) were chosen as the h-line were known as suitable for several hundred microns tall high aspect ratio $\mathrm{SU}-8$ structures. Traditionally, the i-line of $365 \mathrm{~nm}$ has been used for the SU-8 process because of the high absorption of the light and, thereby, the fast process. However, because of the high attenuation from the high absorption of the i-line, the $\mathrm{i}$-line has been challenging to use for the multiple hundreds micron thick SU-8 process. The h-line of $405 \mathrm{~nm}$ has a relatively low light absorption rate over the SU-8, however, it has better transparency suitable for the thick SU-8 process. The expected longer process time can be resolved by increasing the light intensity of the LEDs, which used to be challenging to the conventional mercury-based UV lamp.

The LED has the length, width, and height as $3.4 \mathrm{~mm}$ by $3.4 \mathrm{~mm}$ by $3.4 \mathrm{~mm}$ and the beam angle was $55^{\circ}$. Each LED can handle the current up to $500 \mathrm{~mA}$ with $3.4 \mathrm{~V}$ for $1.15 \mathrm{~W}$. The wavelength of the LED was measured with an optical spectrum analyzer (BLUE-Wave, StellarNet Inc.) as presented in Fig. 3a. Figure 3b shows the 5 by 5 matrix arrangement of the UV LEDs along with the customized waveguides and the corresponding collimating lenses. The peak of the LED shows at $405 \mathrm{~nm}$, and the $50 \%$ intensity bandwidth ranged from 396 to $416 \mathrm{~nm}$, which is a narrow bandwidth for eliminating the need of an expensive optical filter. To test the light exposure characteristics with multiple LEDs as a group, 25 LEDs were mounted on a PCB in 5-by-5 matrix connection so as to control the light intensity, and the on-off switching of the individual LED. A customized optical waveguide and 25 


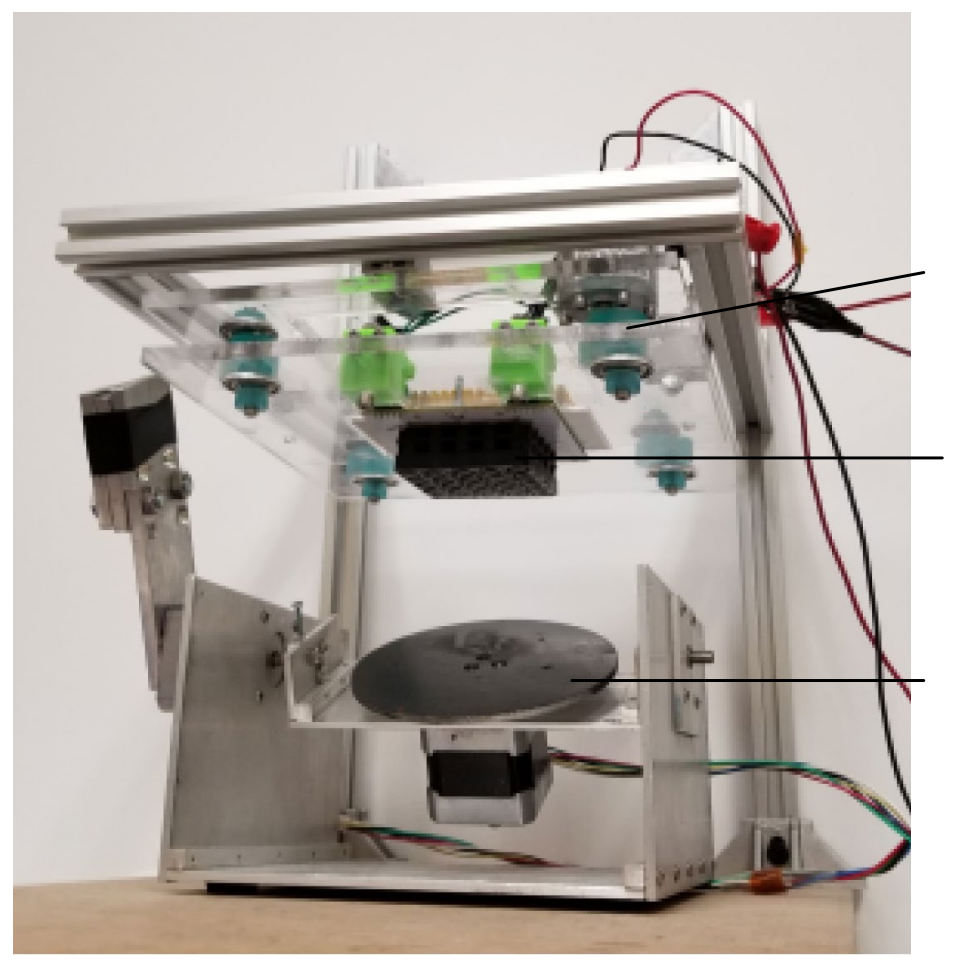

\section{Orbital rotation frame}

5 by 5 LEDs with collimation lens (2" by 2")

Tilt-rotational stage (3")

Fig. 2 System setup

glass lenses were mounted on the PCB. A cabochon cut glass lens (PH PandaHall, Inc.) as shown in Fig. 4a was adopted for light collimation. The lens has a diameter of $10 \mathrm{~mm}$, an offset thickness of $1.3 \mathrm{~mm}$, and a curvature thickness of $2.2 \mathrm{~mm}$ respectively. The cross-sectional view of one unit waveguide is demonstrated in Fig. 4b. The waveguide was designed to position the lens at the focal length for light collimation, block the side light leakage, and derive the air circulation for cooling during the operation. The designed waveguide was 3-D printed using a high-temperature resin, (RS-F2-HTAM-02, Formlabs) and colored with high-temperature black enamel paint (241169, Rust-Oleum).

The completed light source was mounted to an orbital rotational frame where the orbital length was set at 13 $\mathrm{mm}$. The tilt-rotational sample holder was located under the light source where two stepper motors were attached to a disc type sample holder to control tilt and rotational motions. The light switch and the tilt-rotational sample holder were connected to a microcontroller unit (EZ4AXIS, All Motion, Inc.) with a computer. Since the light source has successfully implemented the array of 25 LEDs, the size of the LED can be expanded by adding more LEDs as needed in the future.

\section{Fabrication process}

The fabrication process for 3-D SU-8 photoresist includes sample cleaning, photoresist coating, softbake, UV exposure, post-exposure bake, and development as it is briefly described with the corresponding fabrication result in Fig. 5. Although each process for SU-8 fabrication looks the same as the conventional procedure, there are a few different treatment ways for processing the thick SU-8 photoresist such as $1000 \mu \mathrm{m}$ or thicker. The conventional spin coating process is often not practical because the most amount of the applied photoresist to the substrate used to be a waste when it spins out and more waste for the thicker photoresist process. Furthermore, the achievable thickness by the spin coating method is limited to a couple hundred unless running multiple spinning process. One of the practical solutions for the thick film coating is to use a dry SU-8 film where the pre-manufactured film needs to be attached to the substrate. Although the dry SU-8 film process is convenient and saves the long softbaking time, the preset thickness of the film may not always be well aligned to the thickness that the user requires in various applications. As an alternative way of the thick SU-8 film coating, a weigh and pour method is introduced as shown in Fig. 5A(a). A conventional liquid SU-8 (2025) was weighed and poured on a substrate to coat a final SU-8 thickness of $1200 \mu \mathrm{m}$. 


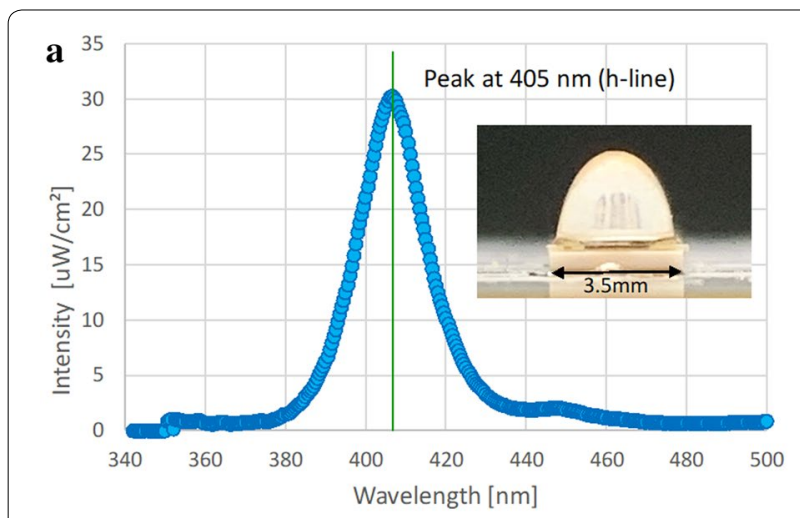

b

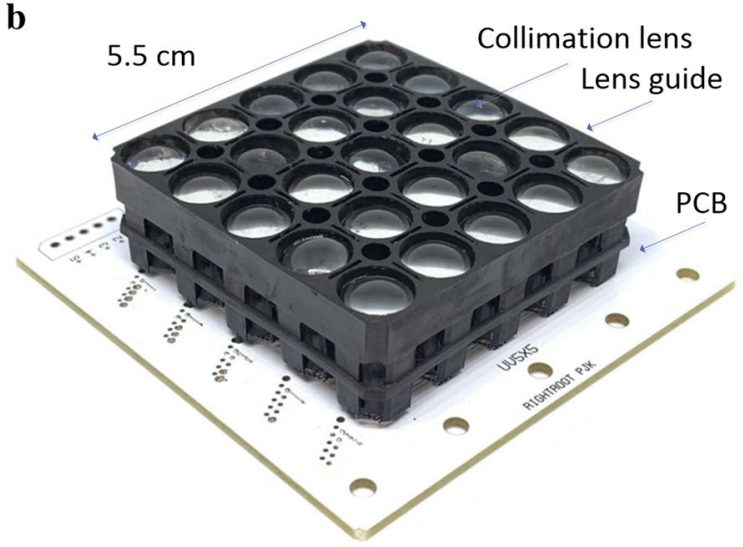

Fig. 3 LED light source, a $405 \mathrm{~nm}$ LED spectrum (inset: the SMD led), b assembled light source

A plastic wall was placed on the substrate before applying the SU-8 to prevent the overflowing. The overflowing barrier wall was 3-D printed using a stereolithography type printer (Form 2, Formlab, Inc.) where the acrylic resin has $100 \%$ fill inside the structure and can bear with $95{ }^{\circ} \mathrm{C}$. The wall is also advantageous for estimating the final SU-8 film thickness during the softbaking process. The substrate later was also served as the photomask as a backside UV exposure scheme. Since the SU-8 2025 includes more solvent (around 75\%) than the photoresist, the initial poured amount of the SU-8 resulted in taller than $1200 \mu \mathrm{m}$, and it gradually reduced as the solvent evaporated. The softbake time and temperature were set at $12 \mathrm{~h}$ and $95{ }^{\circ} \mathrm{C}$ respectively. To demonstrate an example of 3-D exposure scheme, two inclined pillars like ' $\mathrm{V}$ ' shape and a reverse triangle slab were UV-projected through a single micro hole showing in Fig. $5 \mathrm{~A}(\mathrm{~b}-\mathrm{d})$. The 405-nm UV light source was chosen, and the light intensity from the 1 -inch distance was set at $194 \mathrm{~mW} / \mathrm{cm}^{2}$. The first inclined pillar was exposed for $30 \mathrm{~min}$ where the tilt position of the sample holder was set at $60^{\circ}$ as shown in Fig. $5 \mathrm{~A}(\mathrm{~b})$. The sample holder was rotated for $120^{\circ}$ for the second pillar exposure position. The second pillar was exposed for the same energy with the same tilt angle, as shown in Fig. $5 \mathrm{~A}(\mathrm{c})$. The sample holder has again rotated for $120^{\circ}$ again for the exposure of the reverse triangle slab. The continuous UV exposure was performed while the rotation angle oscillates from $+20^{\circ}$ to $-20^{\circ}$ for 60 min as shown in Fig. $5 \mathrm{~A}(\mathrm{~d})$. The sample was bake at $95^{\circ} \mathrm{C}$ for the post exposure bake for an hour and cooled down to room temperature after baking as shown in Fig. 5A(e). Then the samples were dipped into the SU-8 developer solution (MicroChem, Inc.) for 45 min and cleaned with isopropyl alcohol to complete the fabrication, as shown in Fig. $5 \mathrm{~A}(\mathrm{f})$. During the development process, the SU-8 photoresist side was facing down to accelerate the process as the unexposed SU-8 was fell down by gravity during the developing process. The sample was cleaned with isopropyl alcohol (IPA) and dried to complete the fabrication. Figure 5B shows an SEM image of the fabricated $3-\mathrm{D}$ structure where the fabrication process followed from Fig. 5A. The 3-D structure shows two inclined pillars with one reverse triangle shape from one origin. The photomask pattern was a $50-\mu \mathrm{m}$ diameter hole. The diameter of the cross-section of the pillar was larger than the $50 \mu \mathrm{m}$ where it caused by the overexposure dose. The measured height of the 3-D structure was $1200 \mu \mathrm{m}$, which was well aligned with the predicted height.

\section{Result}

The proposed system has been characterized for light collimation, light intensity, and uniformity of the light distribution, and demonstrated several 3-D microstructures using SU-8 photoresist.

Figure 6 shows the result of the light collimation test. 5 LEDs with the collimation lens were placed in one row and a white screen was vertically set to see the light propagation as presented in Fig. 5(left). In a dark room, the 5 LEDs were powered up and projected the light through the white screen. The five columns of lights were observed as shown in Fig. 5(right). The collimation angle was measured at the sidewall of the light propagation column using a software, Image (NIH) and showed the measurement angle within $5^{\circ}$, which is acceptable for the photolithography purpose.

Figure 7 shows the measurement result of the UV-LED light intensity and the associated thermal effect on the LED. As the adjustable intensity of the light source was considered as an important feature for the proposed system, the light intensity was measured via applying different amounts of the current per each LED as shown in Fig. 7a. The maximum allowed current for each LED was indicated as $500 \mathrm{~mA}$, however the maximum applied current in this experiment was limited up to $320 \mathrm{~mA}$, which was noted as a rated current from the manufacturer. The applied current was increased in every $8 \mathrm{~mA}$, and then 


\section{$\mathbf{a}$}

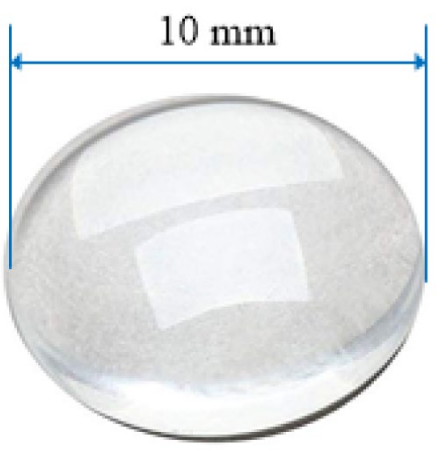

$2.2 \mathrm{~mm}$

$1.3 \mathrm{~mm}$

b

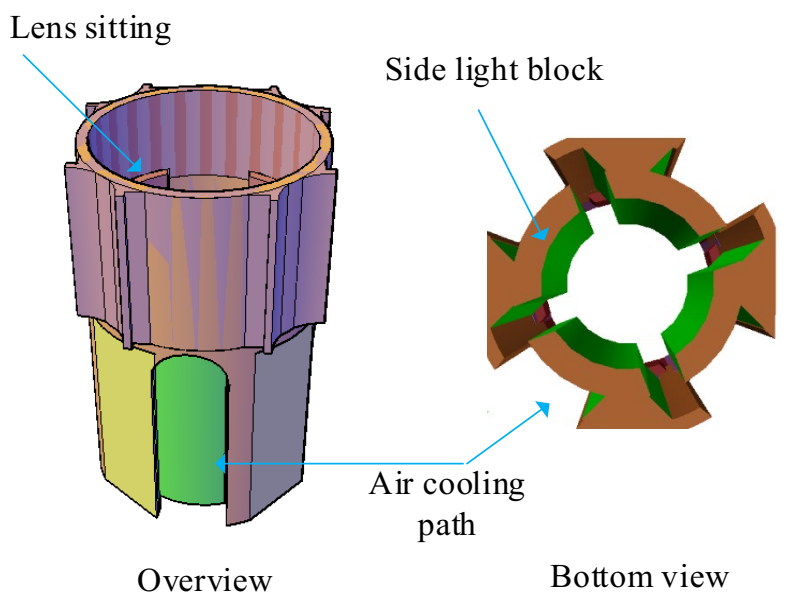

Fig. 4 Designs of $\mathbf{a}$ lens and $\mathbf{b}$ waveguide

the light intensity was measured at the distance of 20 $\mathrm{mm}, 40 \mathrm{~mm}, 60 \mathrm{~mm}$, and $120 \mathrm{~mm}$. At the distance of 20 $\mathrm{mm}$, the light intensity was reached at $200 \mathrm{~mW} / \mathrm{cm}^{2}$ at $200 \mathrm{~mA}$, so did the same at the distance of $40 \mathrm{~mm}$ at 280 $\mathrm{mA}$ where the range of the intensity meter (Model 202, G\&R Labs) was up to $200 \mathrm{~mW} / \mathrm{cm}^{2}$ at the same scale. The light intensity at the distance of $120 \mathrm{~mm}$ still showed above $100 \mathrm{~mW} / \mathrm{cm}^{2}$ which is still high enough for millimeter thick SU-8 process. The distance of $120 \mathrm{~mm}$ also secures for the space of the tilt-rotational sample holder where the plate diameter was around $750 \mathrm{~mm}$. The measured data of the variable light intensity and the different distance data can be a reference later for the programmable light exposure process.

Thermal behavior of the LED was observed while the applied current to each LED was increased up to 320 $\mathrm{mA}$ with every $8 \mathrm{~mA}$ step. The first observation was conducted without any heat management tool. Starting from a room temperature, each LED was heated up to 129.7 ${ }^{\circ} \mathrm{C}$ at the $320 \mathrm{~mA}$. The same test was performed after adding an aluminum heatsink (BNTECHGO, Inc.) to the backside of the UV-LED PCB board. The heat increment was proportional to the applied current amount and the highest temperature was observed at $87.3{ }^{\circ} \mathrm{C}$. Based on the recommended temperature for the LED from the manufacturer, the $87.3^{\circ} \mathrm{C}$ was marginally acceptable. An external fan was added, and the same temperature experiment was conducted. While observing the slow increment of the temperature, the highest temperature was $48^{\circ} \mathrm{C}$ at $320 \mathrm{~mA}$. Assuming that the optical temperature can be different from different models and manufacture, the heat sink and the speed adjustable cooling fan can operate the LEDs at the recommended range of the temperature.

The light source of the system was designed to have an orbital rotation, which is advantageous for programmable LED control as the location of each LED has a regional rotation without changing the position with neighbored LEDs while providing uniform light intensity over the substrate. Figure 8 shows the comparison of the UV-LED 

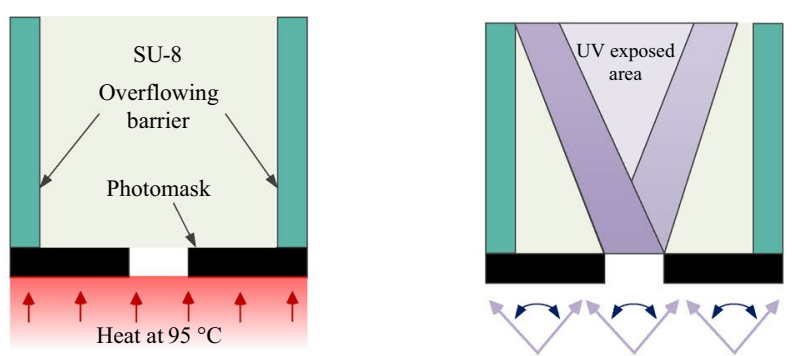

a. SU-8 coating \& Softbake

d. Third exposure: inclined slab
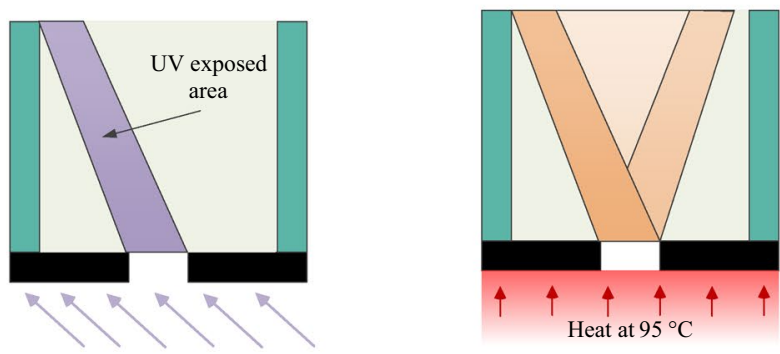

b. First exposure: inclined pillar

e. Post exposure bake
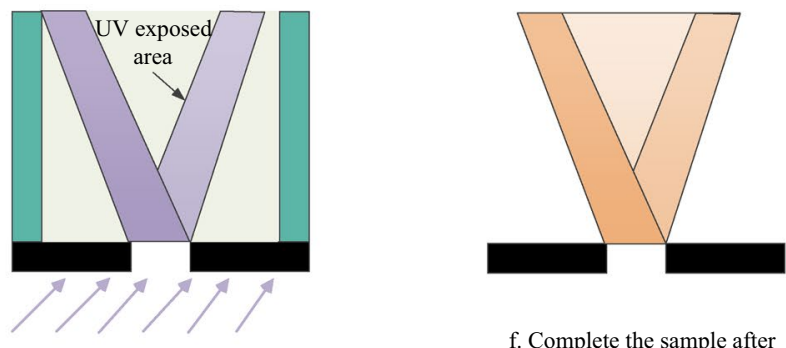

c. Second exposure: inclined pillar

f. Complete the sample after developing

a

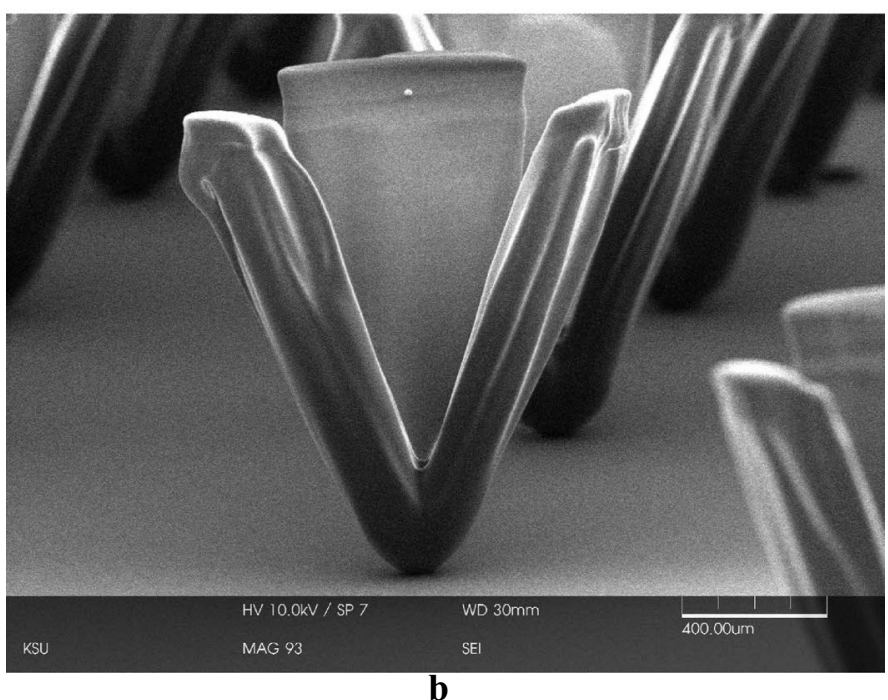

Fig. 5 a Fabrication process and $\mathbf{b}$ the fabricated 3D structure 

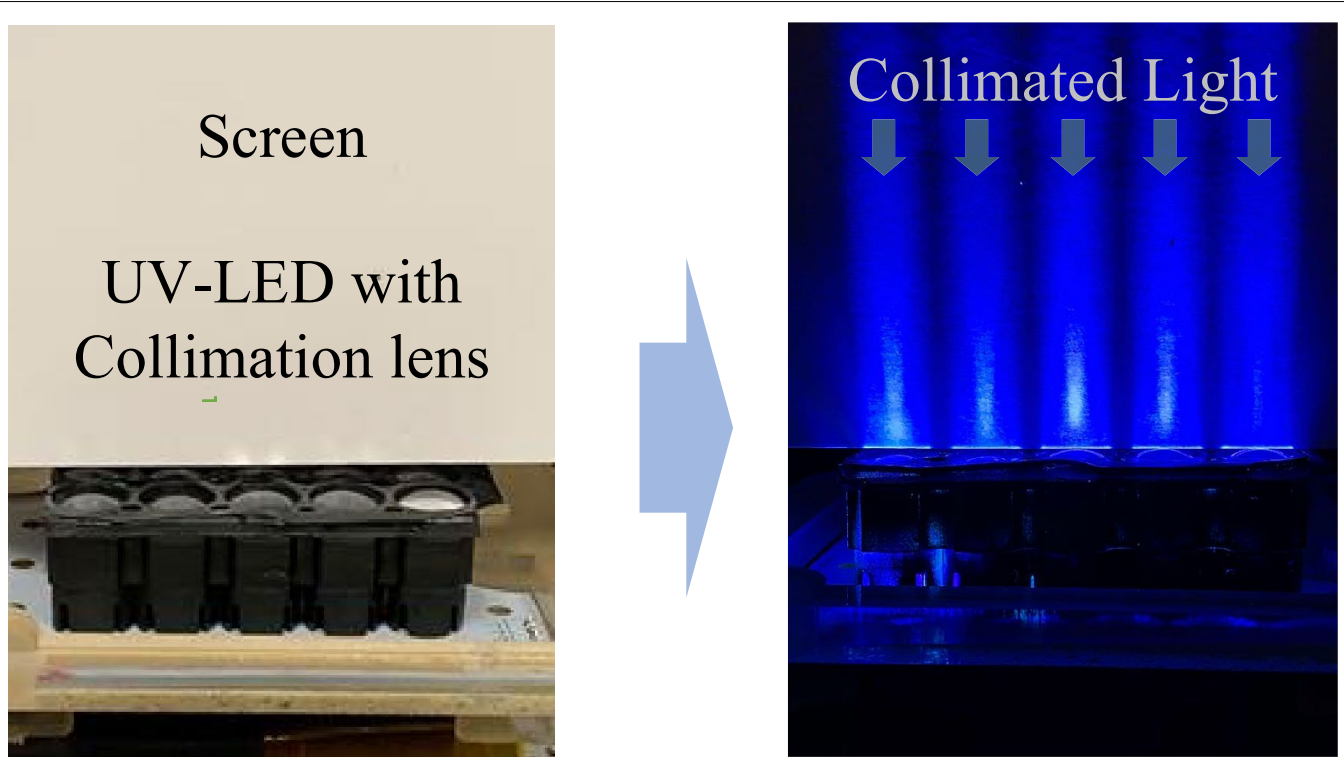

Fig. 6 Light propagation, a waveguide with lens and collimation: The collimation angle was observed with $5^{\circ}$

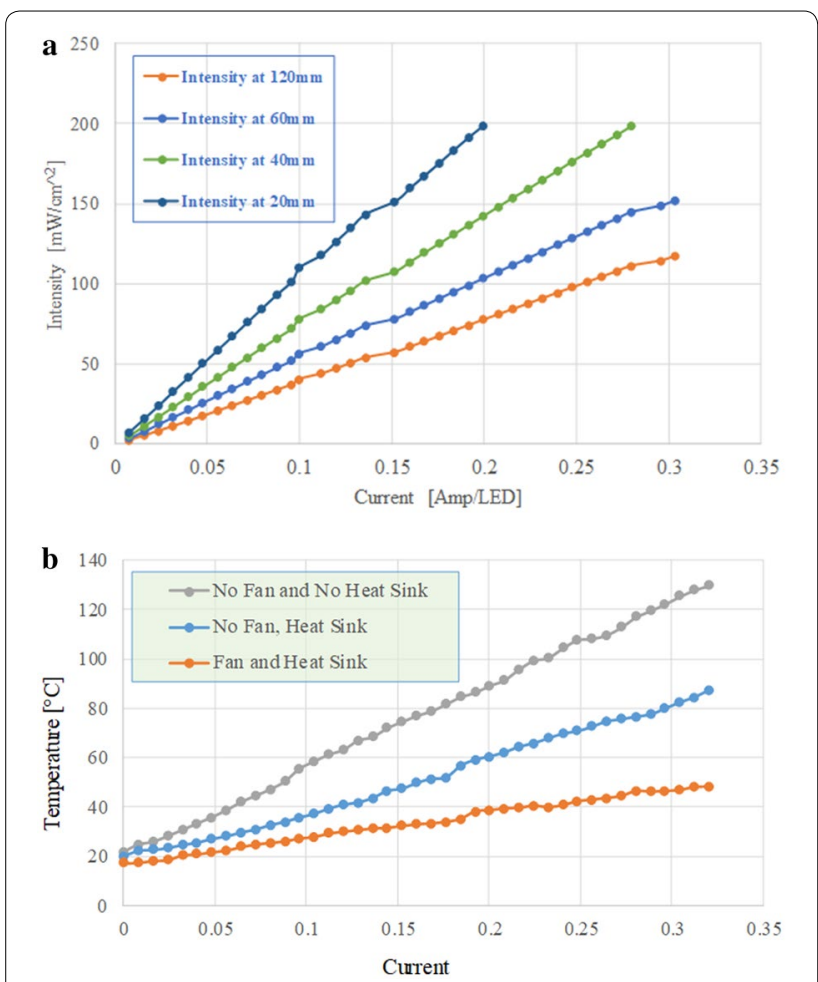

Fig. 7 UV-LED light source characteristics: a light intensity via applied current per led at a different distance, and $\mathbf{b}$ temperature variation over the applied current with different cooling methods

light intensity distribution with and without orbital oration. The 5-by-5 UV-LED light source is designed to rotate with an orbital axis for uniform distribution of the light intensity. The axis length was designed as $13 \mathrm{~mm}$ to cover the entire exposed area. The images were captured using a camera (EOS Rebel T3i, Canon) for manual exposure mode for $8 \mathrm{~s}$. To present and measure the intensity of the light, a thin white screen was placed in front of the UV-LED array. Figure 8a shows the light-on image of the 5-by-5 UV-LED array. Exposed areas were shown in the area where the lenses were placed. High contrast between the exposed area and the non-exposed area was found. Figure $8 \mathrm{~b}$ shows the light exposure image with the orbital rotation. The exposed area was slightly larger than the image shown in Fig. 8a due to the rotation and, as expected, the image shows the uniform distribution of the light exposure area. The captured images of Fig. 8a, b were further analyzed with the intensity contrast through the black line drawn at the center of the image. Using a software, ImageJ, the light intensity contrast profile was plotted as shown in Fig. 8c. The blue dots (no rotation) shows the high-intensity contrast ranging from 0.45 to 0.95 while the red dots (with rotation) show a relatively uniform light distribution.

To provide the same LED intensity from the multiple LEDs, the provided power to each LED can be adjustable. This feature is advantageous because a number of LEDs possibly show a slight difference in the intensity by manufacturing. On the other hand, the system can provide regional high contrast light distribution aiming non-uniform light distribution as needed. For example, micropatterning the photoresist with various height requires locally differentiated light intensity. This advanced feature has great potential as the next generation of 

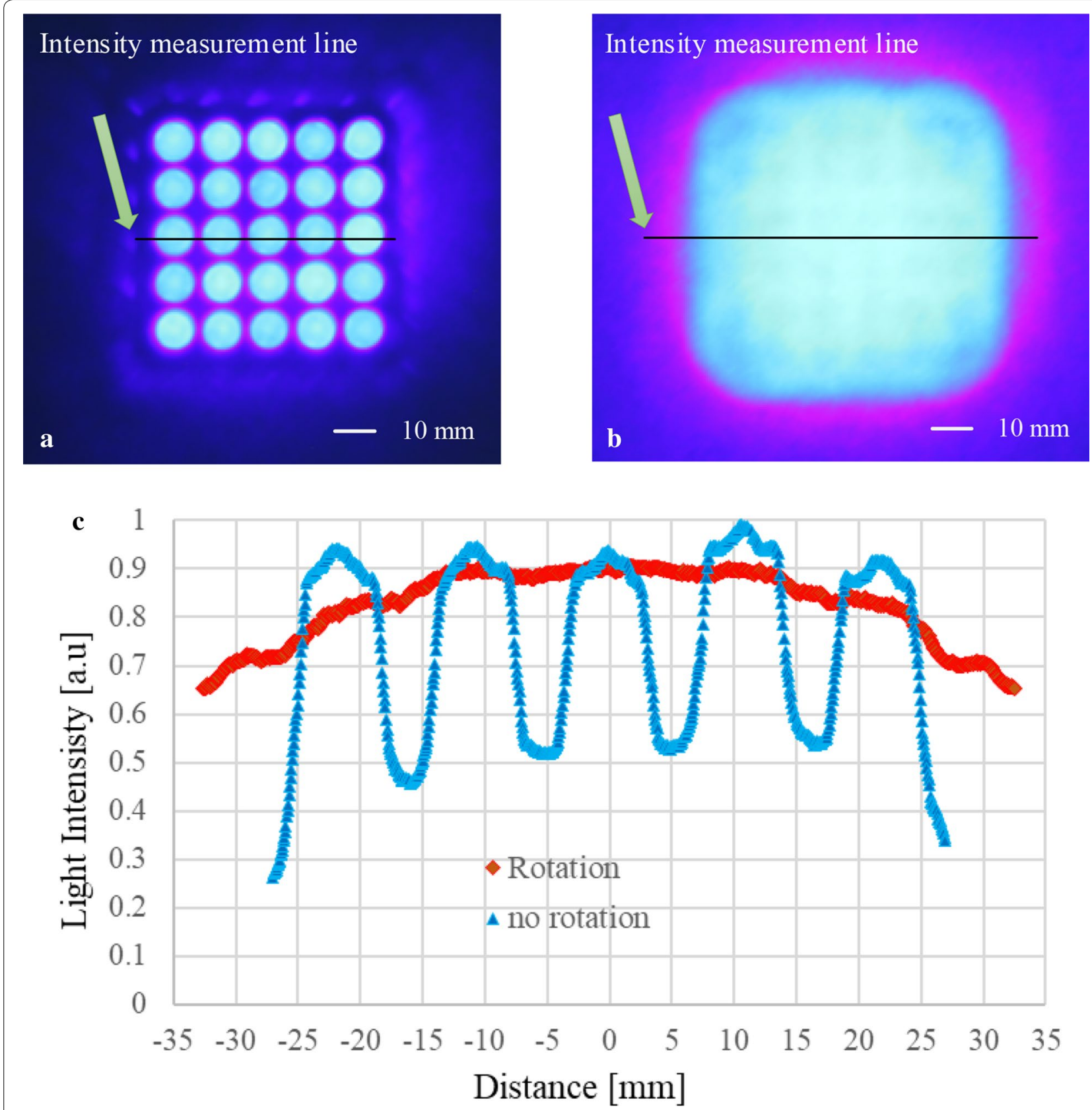

Fig. 8 Light uniformity test result: a the led light source without rotation, $\mathbf{b}$ with rotation, and $\mathbf{c}$ the light intensity distribution graph from (a) and (b)

the microlithography system to create a variety of 3D microstructures.

Figure 9 shows a transparency data of the SU-8 with various thicknesses in the range of $0.5 \mathrm{~mm}$ to $7 \mathrm{~mm}$. In the conventional SU-8 photolithography, the thickness of the SU- 8 has been typically utilized in a few microns to hundreds of microns because of the fabrication challenges such as long exposure time, low UV light intensity, and a long softbake time and incompatibility of a few millimeter thick SU-8 casting. However, the UV-LED with very high intensity can resolve some of the major challenges for the millimeter range SU-8 process. Since lithography is a light exposure process in particularly important with the light propagation through the photoresist, the SU-8's transparency characteristics via UV exposure time in millimeter thick range was conducted. 


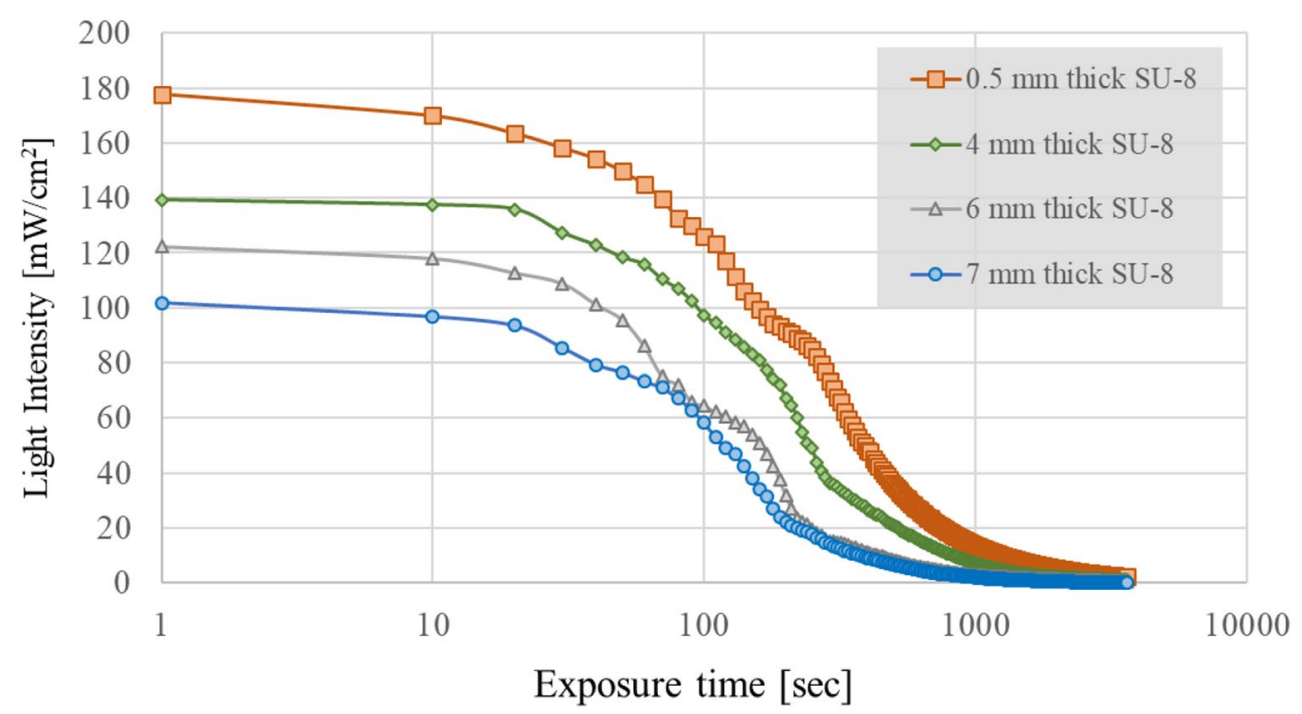

Fig. 9 Through SU-8 Transparency and attenuation tests

In the experiment, the thick SU-8 (SU-8 2025) sample was cast on a clear glass substrate and softbaked at $95^{\circ} \mathrm{C}$ for $10-15 \mathrm{~h}$, depending on the thickness. The baked SU-8 sample was assumed that most solvents were evaporated. The sample was placed underneath of the UV-LED (405 nm) and measured the light intensity at the opposite side of the sample with the intensity meter (Model 202, G\&R Labs). The graph shows that the light intensity maintained for the first $10 \mathrm{~s}$ and started to degrade. Most samples showed a similar attenuation rate of the light intensity in the exposure time range of 10 to 100 as the intensity dropped with 30 to $40 \%$ after 100 s exposure. The light intensity was significantly dropped after $1000 \mathrm{~s}$ (about $17 \mathrm{~min}$ ), where $10 \%$ or lower to the original intensity remained. As the intensity of the UV-LED light source was set at $200 \mathrm{~mW} / \mathrm{cm}^{2}$, the suggested exposure time for the UV-LED is up to $600 \mathrm{~s}(10 \mathrm{~min})$ where the light intensity still shows higher than $10 \mathrm{~mW} / \mathrm{cm}^{2}$ which can still derive the SU-8 crosslinking reaction.

Figure 10 presents some conventional microstructures using the introduced UV-LED systems to shows that the UV-LED systems can fabricate the small feature size patterns as well as high aspect ratio pillars. Figure 10a shows an SEM image of multiple lines. The narrow lines are 3.5 $\mu \mathrm{m}$, and the wide lines are 8 microns, where the patterns were made of SU-8. Since many photopattern sizes with the $365 \mathrm{~nm}$ or $405 \mathrm{~nm}$ lights used to be a few microns or larger, the demonstrated $3.5 \mu \mathrm{m}$ feature size shows good compatibility with the conventional UV lithography uses. Figure 10b shows the high-aspect-ratio SU-8 pillar where the diameter of the pillar was $20 \mu \mathrm{m}$, and the height of the pillar was $700 \mu \mathrm{m}$. The aspect ratio of this pillar is 35 , which used to be hard to fabricate unless the UV light source has high intensity and high light collimation quality. Figure $10 \mathrm{c}$ shows a $3-\mathrm{mm}$ tall SU-8 pillar array. The height of the SU-8 pillar shows extremely tall as compared to the conventional SU-8 structures. The diameter of the pillar was $300 \mu \mathrm{m}$, and therefore the aspect ratio of the pillar shows 10. In this 3-mm tall SU-8 pillar fabrication, a total UV-exposure time of $10 \mathrm{~min}$ was applied at $200 \mathrm{~mW} / \mathrm{cm}^{2}$ where the exposure time is still practical, and yet the extended height of the SU-8 structure can have a great potential for RF/microwave antenna or microfluidic channel applications. Figure 10d shows an array of SU-8 micropillars. The 30- $\mu$ m diameter with 400 $\mu \mathrm{m}$ tall SU-8 pillar was uniformly fabricated to show the batch process. More than 2000 pillars are presented in the SEM image.

Figure 11 shows various 3-D SU-8 structures fabricated using the UV-LED light and the tilt-rotational stage. Figure 11a shows an array of 'chained triangular slab'. An array of a $30-\mu \mathrm{m}$ hole on a chromium glass was utilized as a substrate as well as a backside exposure photomask. $300-\mu \mathrm{m}$ thick SU-8 was coated on the substrate and baked for $95{ }^{\circ} \mathrm{C}$. The tilt-rotation sample holder was programed to continuously move the tilt angle from $-70^{\circ}$ to $+70^{\circ}$ while the rotation angle was fixed. The light intensity was set at $200 \mathrm{~mW} / \mathrm{cm}^{2}$, and the total applied light energy was $480 \mathrm{~J} / \mathrm{cm}^{2}$. Figure $11 \mathrm{~b}$ shows a millimeter-scale $\mathrm{SU}-8$ tripod structure. $1.5-\mathrm{mm}$ thick SU-8 was coated on the chromium glass for the backside exposure. The tilt-rotational sample holder was programmed as follows. The tilt angle was fixed at $70^{\circ}$, and the UV exposure was performed at 

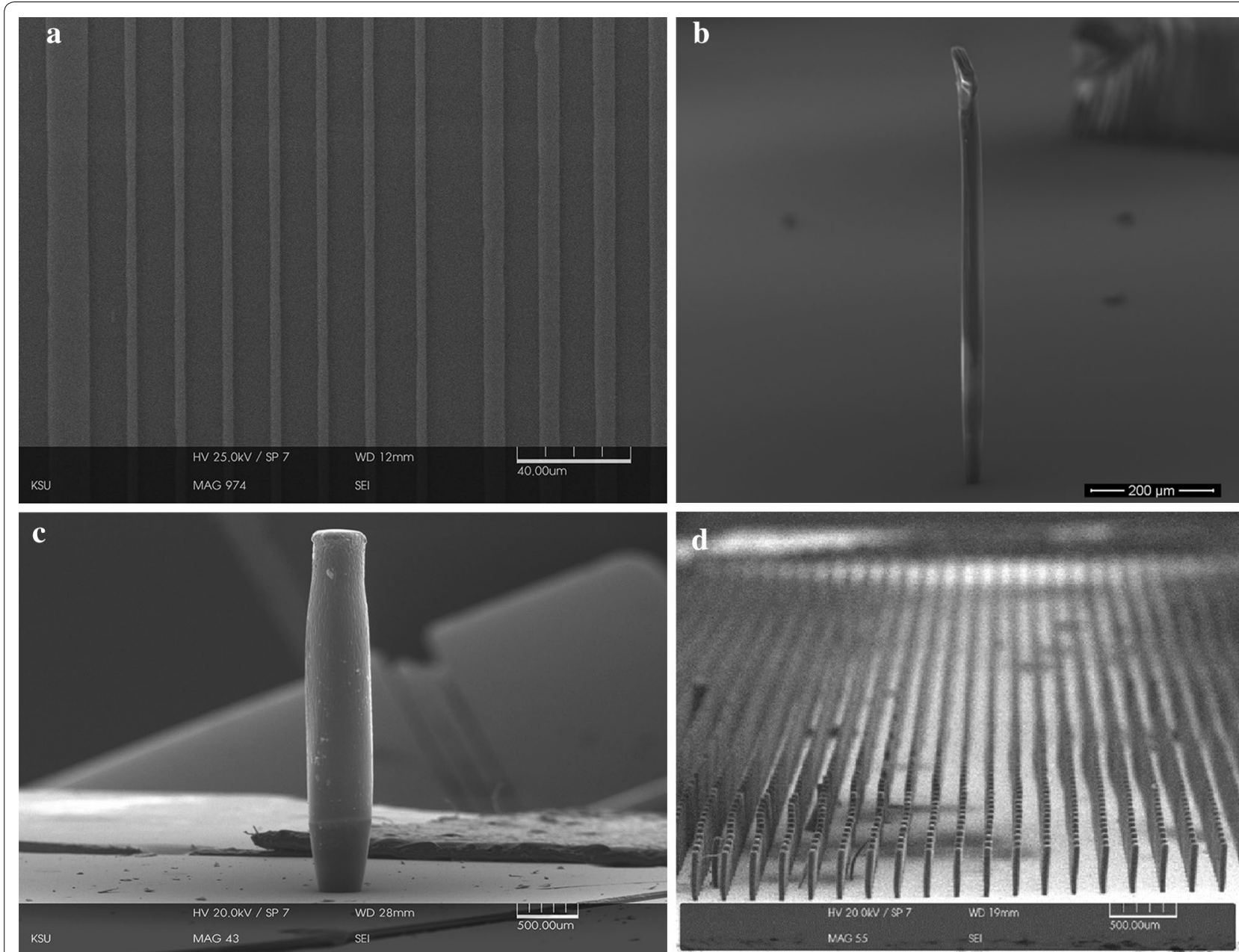

Fig. 10 Small feature size, and high aspect ratio pillar, millimeter tall high aspect ratio pillar: a 3.5 and 8 micron lines, $\mathbf{b}$ high aspect ratio micropillar, c 3-mm tall micropillar, $\mathbf{d}$ more than 2000 micropillar array

the stationary rotation angles of $0^{\circ}, 120^{\circ}$, and $240^{\circ}$ for $360 \mathrm{~J} / \mathrm{cm}^{2}$ respectively. The inclined angle of each pillar was measured as $67^{\circ}$ from the ground, which was well aligned with the prediction from the Snell's law with a refractive index of 1.67 for SU-8. The length of the inclined pillar was measured at $2.2 \mathrm{~mm}$. Figure $11 \mathrm{c}$ shows 3D SU-8 petal structures with different sizes on the same process. The size differences were caused by the different size of the photomask patterns, which would differentiate the applied UV exposure energy. The height of the large petal SU-8 structure shown in Fig. 11c (left) was $1.5 \mathrm{~mm}$ while the height of the small petal SU-8 structure was measured as $200 \mu \mathrm{m}$. The presented 3-D structures have great potential.

\section{Conclusion}

A high-intensity UV-LED lithography system was presented for the fabrication of millimeter tall 3-D microstructures. The presented light intensity showed around $200 \mathrm{~mW} / \mathrm{cm}^{2}$, where the h-line of the UV light is transparent to several millimeter thick SU-8 photoresists enabling millimeter-scale 3-D microlithography. The uniqueness of the 5-by-5 LED array light source includes collimated lenses with customized light waveguide, and the whole light source has an orbital rotation for uniform light distribution as well as regional light on-off/intensity control for various photopatterning capability. The tiltrotational sample holder creates 3-D light traces into the photoresist for 3-D microfabrication.

Since the introduced UV-LED system has highintensity 3-D projectable collimation light for both the conventional photoresist process and the advanced millimeter thick SU-8 process, test microlithography 

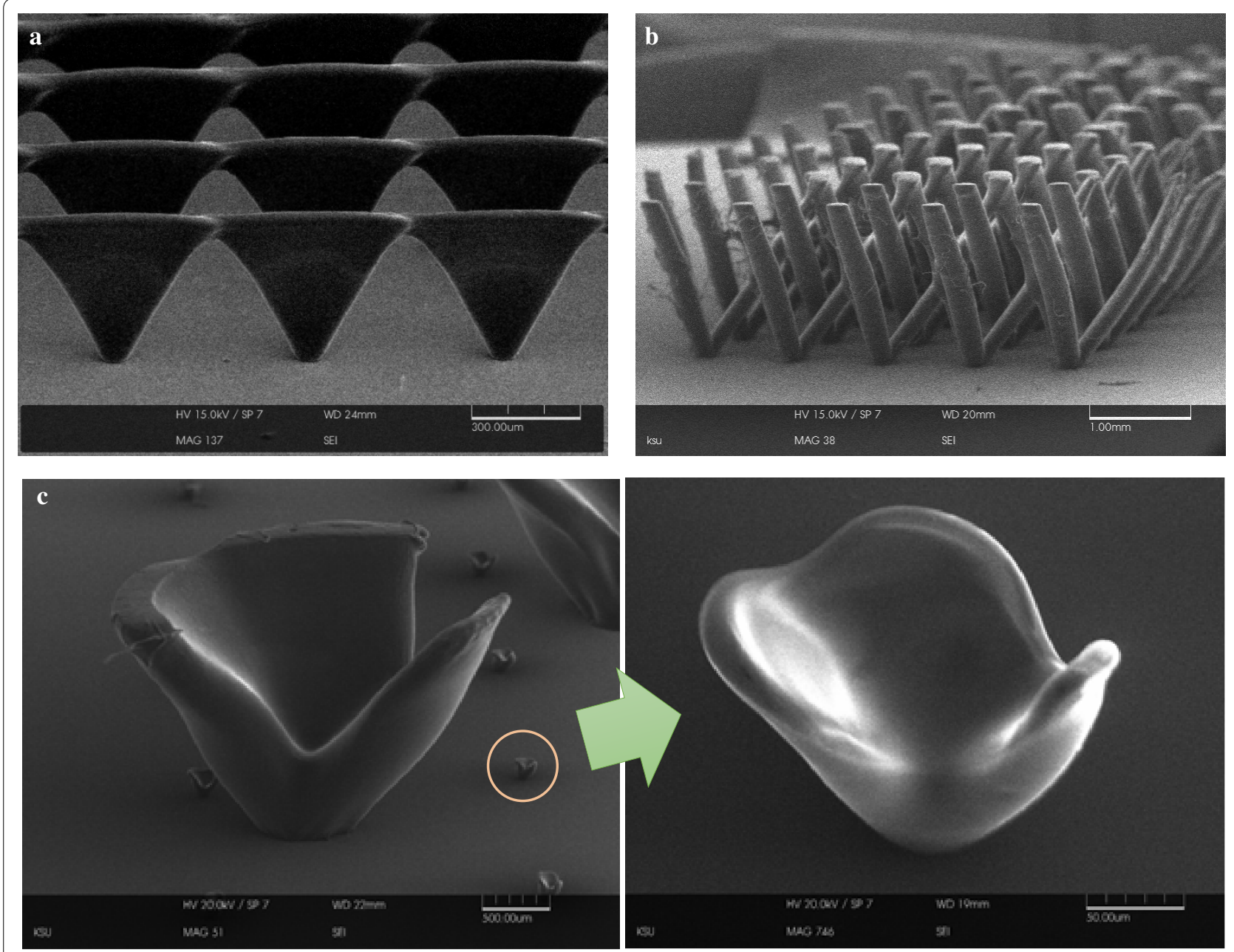

Fig. 11 Unique 3D SU-8 structures: a an array of 'chained triangular slab', b millimeter-tall SU-8 tripod array, c large (1.5 mm tall) and small (200 $\mu \mathrm{m}$ tall) 3D petal SU-8 structure on a single structure

structures including a few micron small feature size of SU-8 patterns, high aspect ratio SU-8 structures, and millimeter tall SU-8 structures were successfully fabricated and demonstrated.

These unique 3-D microstructures with expanded height in millimeter-scale expanded design of photopatternable 3-D microstructures with the highintensity UV-LED exposure process and have great potentials in device fabrication of RF- and Bio-MEMS fields. Also, as the proposed system showed the simplicity of the light source with a relatively small dimension, it can be used as a modular-based light exposure system to create a complex light exposure system for more freedom of 3D microfabrication capability in the future.

\section{Abbreviations}

UV: Ultra-violet; LED: Light emitting diode; CLiPP: Contact liquid photolithographic polymerization; DMD: Digital micromirror display; LCD: Liquid crystal display; RF: Radio frequency; IPA: Isopropyl alcohol; PCB: Printed circuit board.

\section{Acknowledgements}

Authors thank to Samil Tech, Co. LTD for the kind donation of the UV-LED integrated PCB.

\section{Authors' contributions}

JK drafted the manuscript. SFS and JYT conducted and analyzed the light transparency and intensity attenuation data. SFS fabricated the 3-D SU-8 structures. All the authors discussed the proposed method and experimental results. All authors read and approved the final manuscript.

\section{Funding}

This work has been partially supported by an international collaborative research project sponsored by the Korea Institute for Advancement of Technology (KIAT, 2017-52, N058900002) and Samil Tech Co. LTD.

\section{Availability of data and materials}

The datasets supporting the conclusions of this article are included within the article. 


\section{Competing interests}

The authors declare that they have no competing interests.

Received: 28 October 2019 Accepted: 13 April 2020

Published online: 20 April 2020

\section{References}

1. Han M, Lee W, Lee S-K, Lee SS (2003) Fabrication of 3D microstructures with inclined/rotated UV lithography. In: The sixteenth annual international conference on micro electro mechanical systems, MEMS-03 Kyoto, IEEE, pp 554-557

2. Hutchison JB, Haraldsson KT, Good BT, Sebra RP, Luo N, Anseth KS, Bowman CN (2004) Robust polymer microfluidic device fabrication via contact liquid photolithographic polymerization (CLiPP). Lab Chip 4:658. https://doi.org/10.1039/b405985a

3. Ikuta K, Hirowatari K (1993) Real three dimensional micro fabrication using stereo lithography and metal molding. In: Proceedings IEEE micro electro mechanical systems, IEEE, pp 42-47

4. Jiang G, Baig S, Wang MR (2012) Prism-assisted inclined UV lithography for 3D microstructure fabrication. J Micromech Microeng 22:085022. https://doi.org/10.1088/0960-1317/22/8/085022

5. Kim J (2010) Advanced multidirectional UV lithography for three dimensional (3-D) micro-/nano structures. State University of New York, Buffalo

6. Kim J, Al Thuwaini H, Almuslem M (2018) Photolithography of SU-8 microtowers for a 100-turn, 3-D toroidal microinductor. Micro Nano Syst Lett 6:14. https://doi.org/10.1186/s40486-018-0076-z

7. Kim J, Allen MG, Yoon Y-K (2011) Computer-controlled dynamic mode multidirectional UV lithography for 3D microfabrication. J Micromech Microeng 21:035003. https://doi.org/10.1088/0960-1317/21/3/035003
8. Kim J JK' (2019) UV-LED lithography for millimeter-tall high-aspect ratio $3 \mathrm{~d}$ structures. Institute of electrical and electronics engineers (IEEE), pp 100-103

9. Kim J, Yoon Y-K, Allen MG (2016) Computer numerical control (CNC) lithography: light-motion synchronized UV-LED lithography for 3D microfabrication. J Micromech Microeng 26:035003. https://doi. org/10.1088/0960-1317/26/3/035003

10. Kim J, Yun T-S, Jee H, Yoon YK (2009) Adjustable refractive index method for complex microstructures by automated dynamic mode multidirectional UV lithography. In: 2009 IEEE 22nd international conference on micro electro mechanical systems, IEEE, pp 733-736

11. Nakamura K, Kubota H, Nakada A, Inokuchi T, Kosaka K (2002) Development of reticle-free exposure method with LCD projection image. In: Kawahira H (ed) International Society for Optics and Photonics, p 737

12. Peterman MC, Huie P, Bloom DM, Fishman HA (2003) Building thick photoresist structures from the bottom up. J Micromech Microeng 13:380-382. https://doi.org/10.1088/0960-1317/13/3/305

13. Yang $W$, Yu H, Wang $Y$, Liu L (2015) Fabrication of microstructures using the DMD-based modulating projection printing method. In: 10th IEEE international conference on nano/micro engineered and molecular systems, IEEE, pp 625-629

14. Yoon Y-K, Park J-H, Allen MG (2006) Multidirectional UV lithography for complex 3-D MEMS structures. J Microelectromech Syst 15:1121-1130. https://doi.org/10.1109/JMEMS.2006.879669

\section{Publisher's Note}

Springer Nature remains neutral with regard to jurisdictional claims in published maps and institutional affiliations.

\section{Submit your manuscript to a SpringerOpen ${ }^{\circ}$ journal and benefit from:}

- Convenient online submission

- Rigorous peer review

- Open access: articles freely available online

- High visibility within the field

Retaining the copyright to your article

Submit your next manuscript at $\boldsymbol{\nabla}$ springeropen.com 